\title{
SPLITTING CRITERIA FOR MODULAR LATTICES
}

\section{AILEEN HOSTINSKY}

Within the framework of a complete modular lattice two types of criteria for uniform splitting are developed in the first part of this note. One deals with the concept of an $\eta$-automorphic element, and, under the hypothesis that $x$ is $\eta$-automorphic, it is shown that an endomorphism $\eta$ is a uniformly splitting endomorphism of $p / 0$ if and only if $\eta$ induces a uniformly splitting endomorphism of $p / x$. The other type is concerned with the existence of a set of elements which are uniformly split by an endomorphism. In the last section application of this theory of splitting gives a further connection between homomorphisms and direct decompositions. The work is based upon and extends certain previous investigations $[2 ; 3 ; 4]$ by the author into this problem.

Earlier (see [1]) in group theory criteria similar to the first type have been developed for the existence of complements which are normal subgroups and have been used in studying extension types. The present paper generalizes certain of these results by lattice-theoretical methods not used in [1].

1. Preliminaries. The fundamental properties of endomorphisms, uniform splitting, and direct decompositions upon which this paper is based have been developed in [2] and [3]. The terminology and notation of these papers will be used here. A few of the needed facts are now summarized. The algebraic system is a complete modular lattice $L$ satisfying the additional Property $\left({ }^{*}\right): a \sum_{\alpha} p_{\alpha}=\sum_{\alpha} a p_{\alpha}$ for $a$ and the ascending chain $p_{\alpha}$ elements of $L$. A mapping $\eta$ is a homomorphism of the quotient $p / p^{\prime}$ in $L$ upon $q / q^{\prime}$ in $K$ (a system satisfying the same postulates as $L$, or $L$ itself) if $\eta$ maps $x / p^{\prime}$ for every $x$ satisfying $p^{\prime} \leqq x \leqq p$ upon $x \eta / q^{\prime}$, where $q^{\prime} \leqq x \eta \leqq q$, so that the following requirements hold:

(1) $\left(\sum_{x} x\right) \eta=\sum_{x}(x \eta)$.

(2) If $q^{\prime} \leqq z \leqq x \eta$, then there exists an element $z^{\prime}$ with $p^{\prime} \leqq z^{\prime} \leqq x$ such that $z^{\prime} \eta=z$.

(3) If $x \eta=y \eta$, then there exist elements $x^{\prime}$ and $y^{\prime}\left(p^{\prime} \leqq x^{\prime} \leqq p\right.$ and $\left.p^{\prime} \leqq y^{\prime} \leqq p\right)$ such that $x+x^{\prime}=y+y^{\prime}$ and $x^{\prime} \eta=y^{\prime} \eta=q^{\prime}$.

A homomorphism $\eta$ of $p / 0$ upon $p \eta / 0$ is said to be an endomorphism of $p / 0$ if $p \eta \leqq p$. The kernel $k(\eta)$ of this endomorphism is the sum of all elements which $\eta$ maps upon 0 , and the radical $r(\eta)$ is defined as

Received by the editors October 8, 1958 and, in revised form, April 21, 1959. 
$\sum_{i=1}^{\infty} k\left(\eta^{i}\right) . x$ is $\eta$-admissible if $x \eta \leqq x$. The usual homomorphism and isomorphism theorems hold.

The element $c$ is a complement of the endomorphism $\eta$ of $p / 0$ if $p=r(\eta)+c, c r(\eta)=0$ (that is, $p=c \oplus r(\eta)$ ), and $c \eta=c$. If there exists a complement of $\eta$, then $\eta$ is a splitting endomorphism of $p / 0$ or is said to split $p$. If $\eta$ induces a splitting endomorphism of $x / 0$ for every $\eta$-admissible $x, \eta$ is a uniformly splitting endomorphism of $p / 0$. The element $x$ is $\eta$-automorphic if $x \eta \leqq x \leqq p$ and if $y \eta \leqq y \leqq x$ implies $y \eta=y$.

(a) If $\eta$ is a homomorphism of $p / p^{\prime}$ upon $q / q^{\prime}$ and if $p^{\prime} \leqq x \leqq p$, then

(i) $x \eta=q^{\prime}$ if and only if $x \leqq k(\eta)$.

(ii) $x \eta=y \eta$ if and only if $x+k(\eta)=y+k(\eta)$ [2, Lemma 1, p. 333].

(b) If the endomorphism $\eta$ of $p / 0$ splits $p$ uniformly, then the complement $c(\eta)$ of $\eta$ is uniquely determined, $\eta$-automorphic, and equal to the sum of all $\eta$-automorphic elements [2, Theorem 2, p. 337].

(c) If $\eta$ splits $p$ uniformly with complement $c(\eta)$ and if $x$ is an $\eta$-admissible element, then $x c(\eta)$ is the uniquely determined complement of the endomorphism which $\eta$ induces in $x / 0$ [2, Corollary, $\mathrm{p}$. 338 ].

(d) If $t$ is $\eta$-automorphic, $\operatorname{tr}(\eta)=0$ [2, Lemma 1, p. 336].

2. $\eta$-automorphic elements and splitting. If $x$ is $\eta$-automorphic, the endomorphism $\eta$ of $p / 0$ induces an endomorphism of $p / x$. In this section relationships between splitting endomorphisms of $p / 0$ and of $p / x$ are considered.

Lemma 1. If $x$ is $\eta$-automorphic, then $x r(\eta)=0$.

PROOF. If $x$ is $\eta$-automorphic, it follows from (d) that $\operatorname{xr}(\eta)=0$.

Lemma 2. If $x$ is $\eta$-automorphic, then $x \oplus r(\eta)$ is the radical of the endomorphism induced by $\eta$ in $p / x$.

Proof. Since $x \eta=x$, the endomorphism induced by $\eta$ in $p / x$ takes $z$ into $z \eta$. Let $y$ be the kernel of the endomorphism which $\eta^{i}$ induces in $p / x$. Then, by use of (a), it follows that

$$
y \eta^{i}=x=x \eta^{i} \text { and } y \leqq y+k\left(\eta^{i}\right)=x+k\left(\eta^{i}\right) .
$$

Since $\left[x+k\left(\eta^{i}\right)\right] \eta^{i}=x \eta^{i}=x, x+k\left(\eta^{i}\right)$ is contained in the kernel, and hence $y=x+k\left(\eta^{i}\right)$. From the definition of the radical and Lemma 1, the radical of the endomorphism induced by $\eta$ in $p / x$ is then $x \oplus r(\eta)$.

THEOREM 1. Let $x$ be $\eta$-automorphic. Then $c$ is a complement of $\eta$ if and only if $x \leqq c$ and $c$ is a complement of the endomorphism induced by $\eta$ in $p / x$. 
Proof. Assume first that $c$ is a complement of $\eta$ and let $y_{i}$ $=x\left(c+k\left(\eta^{i}\right)\right)$. Then

$$
y_{i} \eta \leqq(x \eta)\left[\left(c+k\left(\eta^{i}\right)\right) \eta\right]=x\left(c+k\left(\eta^{i}\right) \eta\right) \leqq x\left(c+k\left(\eta^{i}\right)\right)=y_{i},
$$

since $x$ is $\eta$-automorphic and $c$ is a complement. Thus, $y_{i} \eta \leqq y_{i} \leqq x$, an $\eta$-automorphic element, implies that $y_{i} \eta=y_{i}$. Therefore,

$$
y_{i}=y_{i} \eta^{i} \leqq\left(c+k\left(\eta^{i}\right)\right) \eta^{i}=c \eta^{i}=c .
$$

Since $y_{i} \leqq x$ also, $x c \leqq x\left(c+k\left(\eta^{i}\right)\right)=y_{i} \leqq x c$. Hence, for all $i$,

$$
x\left(c+k\left(\eta^{i}\right)\right)=x c .
$$

Furthermore,

$$
\begin{aligned}
x & =x p=x(c+r(\eta))=x\left(c+\sum_{i=1}^{\infty} k\left(\eta^{i}\right)\right) \\
& =x\left(\sum_{i=1}^{\infty}\left(c+k\left(\eta^{i}\right)\right)\right)=\sum_{i=1}^{\infty} x\left(c+k\left(\eta^{i}\right)\right)=x c .
\end{aligned}
$$

(The last two equalities follow from $\left({ }^{*}\right)$ and (1) respectively.) Thus $x \leqq c$.

By Lemma 2, $x \oplus r(\eta)$ is the radical of the endomorphism induced by $\eta$ in $p / x$. The modular law and $x \leqq c$ imply that $c[x+r(\eta)]$ $=x+c r(\eta)=x$. Also, $c+[x+r(\eta)]=c+r(\eta)=p$, and $c \eta+x=c$. Thus $c$ is a complement of the endomorphism induced by $\eta$ in $p / x$.

Conversely, assume $x \leqq c$ and $c$ is a complement of the endomorphism induced by $\eta$ in $p / x$. Since the product of the complement and radical is $x, x=c[x+r(\eta)]=x+c r(\eta)$, by the modular law. Thus $c r(\eta) \leqq x$, and

$$
\operatorname{cr}(\eta)=\operatorname{cr}(\eta) x=0,
$$

by Lemma 1 . Since $x \leqq c$ and the sum of radical and complement is $p$,

$$
p=c+[x+r(\eta)]=c+r(\eta) .
$$

In order that $c$ be a complement of $\eta$ it remains to show that $c \eta=c$. This fact is evident since $x=x \eta \leqq c \eta$ gives $c=c \eta+x=c \eta$.

THEOREM 2. Let $x$ be $\eta$-automorphic. Then $\eta$ is a uniformly splitting endomorphism of $p / 0$ if and only if $\eta$ induces a uniformly splitting endomorphism of $p / x$.

Proof. Assume that $\eta$ splits $p$ uniformly with complement $c(\eta)$. Then $y c(\eta)$ is the complement of the splitting endomorphism which $\eta$ induces in $y / 0$ for $\eta$-admissible $y$ (by (c)). From Theorem 1 it fol- 
lows that $y c(\eta)$ is a complement of the endomorphism induced by $\eta$ in $y / x$, and hence $\eta$ induces a uniformly splitting endomorphism of $p / x$.

Conversely, assume that there exists a complement $d$ of the endomorphism induced in $y / x$ for $\eta$-admissible $y$. Hence $d[x+y r(\eta)]$ $=x \leqq d$. Thus, by Theorem $1, d$ is a complement of the endomorphism induced by $\eta$ in $y / 0$, and $\eta$ is uniformly splitting.

REMARK 1. It is of interest to note in considering the special case of an induced endomorphism of $p / r(\eta)$ that the assumption that $r(\eta)$ is $\eta$-automorphic implies that $r(\eta)=r(\eta) r(\eta)=0$, by (d).

REMARK 2. In [1] the concept of almost periodic endomorphism is used. Here the endomorphism $\eta$ of $p / 0$ could be defined to be almost periodic on $x / 0$ if $x \eta \leqq x \leqq p$ and if for every $y \leqq x$ there exists a positive integer $j$ (depending upon $y$ ) such that $y=y \eta^{j}$. It is desirable to note that if $\eta$ is almost periodic on $x / 0$, then $x$ is $\eta$-automorphic. The proof is as follows: Let $\eta$ be almost periodic on $x / 0$. Then $x \eta \leqq x \leqq p$, and if $y \eta \leqq y \leqq x$, there exists a positive integer $j$ such that $y=y \eta^{j}$. Since $\eta$ is order preserving, $y \geqq y \eta \geqq y \eta^{2} \geqq \cdots \geqq y \eta^{j}=y$. Hence $y=y \eta$, and $x$ is $\eta$-automorphic. The results of this section, while analogous to results in [1], have been obtained by assuming $x$ to be $\eta$-automorphic, and in some cases the proof is simpler than by using almost periodicity directly. The author wishes to thank the referee for his suggestion of this change in hypothesis with its resulting simplifications.

3. The existence of a set for uniform splitting. A necessary and sufficient condition for uniform splitting is obtained in the following theorem.

THEOREM 3. An endomorphism $\eta$ of $p / 0$ is uniformly splitting if and only if there exists a set $\Phi$ of elements $x \leqq p$ such that

(i) $x \in \Phi$ implies $x \eta \leqq x$ and $\eta$ splits $x$ uniformly,

(ii) $\left\{z_{\alpha}\right\} \subseteq \Phi$ implies that there exists $z \in \Phi$ such that $z \geqq \sum_{\alpha} z_{\alpha}$, and

(iii) $q \leqq p$ and $q \eta \leqq q$ imply $q=\sum_{x \in \Phi} x$, for $x \leqq q$.

Proof. Assume the existence of $\Phi$ and let $y$ be such that $y \eta \leqq y \leqq p$. Then, by (iii), $y=\sum_{x \in \Phi} x$. Thus, by (ii), there exists an element $z$ in $\Phi$ such that $z \geqq y$. Hence, by (i), $\eta$ splits $z$ uniformly, and consequently $\eta$ splits $y$ and $\eta$ is a uniformly splitting endomorphism of $p / 0$.

Assume, conversely, that $\eta$ is uniformly splitting and let $\Phi$ consist of all $\eta$-admissible elements included in $p$. Then

(1) If $x$ is in $\Phi, x \eta \leqq x$ and $\eta$ splits $x$ uniformly.

(2) $z=p$ satisfies condition (ii). 
(3) If $q \eta \leqq q \leqq p$, then $q$ is in $\Phi$ and thus is the sum of elements in $\Phi$ (namely itself).

4. Direct decompositions and homomorphisms. On the basis of the preceding results it is now possible to obtain lattice analogues of Theorems 1 and 2 of $\$ 3$ of [1]. Proofs, which depend largely upon properties of complements and decomposition endomorphisms, are omitted, since they can be obtained in essentially the same manner as in [1]. Necessary modifications in cases where lattice methods were not used to obtain decompositions can be made without great difficulty.

In order to study various elements of $L$ which include $p$, the following definition is useful.

Definition 1 . If $q \geqq p$ and $h \geqq p$, the homomorphism $\eta$ of $q / 0$ upon $h / 0$ is strongly automorphic if $x \eta=x$ for every $x \leqq p$.

Application of the results of $\$ 2$ necessitates imposing the following condition:

(A). For $q \geqq p, q$ is said to satisfy (A) if every strongly automorphic endomorphism of $q / 0$ is a splitting endomorphism.

THEOREM 4. If $q \geqq p$ and $h \geqq p$ and if (A) is satisfied by $q$, then there exist a strongly automorphic homomorphism of $q / 0$ upon $h / 0$ and $a$ strongly automorphic homomorphism of $h / 0$ upon $q / 0$ if and only if there exist direct decompositions

$$
q=q^{\prime} \oplus q^{\prime \prime} \quad \text { and } \quad h=h^{\prime} \oplus h^{\prime \prime}
$$

of $q$ and $h$ such that $p \leqq q^{\prime}$ and there exists an isomorphism of $q^{\prime} / 0$ onto $h^{\prime} / 0$ which leaves invariant every element included in $p$.

Definition 2. The element $q \geqq p$ is a minimal element including $p$ (or, is minimal for $p$ ) if $q=q^{\prime} \oplus q^{\prime \prime}$ and $p \leqq q^{\prime}$ imply that $q=q^{\prime}$ and $q^{\prime \prime}=0$.

Theorem 5. Suppose that $q \geqq p$ and that

$$
q=a^{\prime} \oplus b^{\prime}=a^{\prime \prime} \oplus b^{\prime \prime}
$$

are direct decompositions of $q$ such that $a^{\prime}$ and $a^{\prime \prime}$ are minimal for $p \neq 0$ and $a^{\prime}$ and $a^{\prime \prime}$ satisfy (A). Then

(i) there exists an isomorphism of $a^{\prime} / 0$ onto $a^{\prime \prime} / 0$ which leaves invariant every $x \leqq p$.

(ii) there exists an isomorphism of $b^{\prime} / 0$ onto $b^{\prime \prime} / 0$.

(iii) $q=a^{\prime} \oplus b^{\prime \prime}=a^{\prime \prime} \oplus b^{\prime}$.

It may be of interest to note similarities between the decompositions of Theorem 5 and those of the Refinement Theorems of [3]. 


\section{REFERENCES}

1. R. Baer and C. Williams, Splitting criteria and extension types, Bull. Amer. Math. Soc. vol. 55 (1949) pp. 729-743.

2. L. A. Hostinsky, Endomorphisms of lattices, Duke Math. J. vol. 18 (1951) pp. $331-342$.

3. —_ Direct decompositions in lattices, Amer. J. Math. vol. 73 (1951) pp. 741755.

4. - Loewy chains and uniform splitting of lattices, Proc. Amer. Math. Soc. vol. 5 (1954) pp. 315-319.

The Pennsylvania State University

\section{FINITE DIMENSIONAL CENTRAL DIVISION ALGEBRAS}

\section{S. A. AMITSUR}

1. Introduction. Let $D$ be a division algebra over its center $C$. Let $D[x]$ be the ring of all polynomials in a commutative indeterminate $x$ with coefficients in $D$. The present note contains a characterization of the division algebras $D$ which are finite over their center with the aid of the polynomial ring $D[x]$.

One characterization is the following:

Theorem 1. $(D: C) \leqq n^{2}<\infty$ if and only if every primitive homomorphic image of $D[x]$ is a complete matrix ring $A_{h}, h \leqq n$ over a division ring $A$.

With this result at our hand we utilize recent results on pivotal monomials to show:

Theorem 2. ( $D: C) \leqq n^{2}<\infty$ if and only if for any two polynomials $f(x), g(x) \in D[x]$ the polynomials $1-g(x) f^{n}(x)$ and $f(x)$ do not have a nontrivial left common divisor.

The fact that Theorem 2 gives a characterization of the finite dimensional algebras, means that:

Corollary 1. If $(D: C)>n^{2}$ then $D[x]$ contains two polynomials $g(x), f(x)$ such that $1-g(x) f^{n}(x), f(x)$ possess a nontrivial left common divisor. (Similar result for right divisors.)

We conclude the paper with some remarks on left and right principal ideal rings with primitive images which are finite dimensional matrix rings of bounded degree over division rings.

Received by the editors May 5, 1959. 